\title{
Parallel Computing on the Preheating and Commissioning of Hot Oil Pipelines
}

\author{
Yutian Li, Changchun Wu, Yang Yu \\ Beijing Key Laboratory of Urban Oil and Gas Distribution Technology, China University of Petroleum-Beijing, Changping \\ District, Beijing 102249 PR. China \\ lyt18@163.com,wuchangchun@vip.sina.com,yyogst@163.com
}

\begin{abstract}
In recent years, the numerical simulation method has been widely applied in hydraulic and thermal calculations for hot oil pipelines. Based on the governing equations of fluid flow in hot oil pipelines, a new parallel numerical simulation method for the preheating and commissioning of hot oil pipelines is put forward. From the heat transfer mechanism of hot oil pipelines, a mathematical model on the preheating and commissioning processes of the pipelines is developed. The pipelines and their surrounding soil are discredited by a two-level model, which are the pipeline discretization and the cross-section spatial discretization, then the thermodynamic characteristic line method and the finite volume method were used respectively to discrete the governing equations. Compared with the traditional numerical method, this method can better cope with the sharp thermal transient, during the preheating and commissioning of hot oil pipelines. To improve the computational efficiency of the numerical simulation method, a parallel computing method and the matrix method are applied.

Index Terms - parallel computing, numerical simulation, oil pipeline, preheating, commissioning
\end{abstract}

\section{Introduction}

High pour-point crude and high viscous crude are usually transported by hot oil pipelines (Fig.1) by heating the crude oil in the pipes, the oil viscosity, the friction loss and the power consumption can be reduced, and the wax crystal precipitation can be prevented at the same time. Before the crude pipeline is put into operation, the pipe temperature is equal to the surrounding soil temperature which has great temperature difference with the crude oil injected into the pipeline. As a result, the heat flow between oil and surrounding soil is very intense, and the crude may loss temperature very quickly, and it may cause a rise in viscosity and wax crystal precipitation. All of these will bring threats to the safe operation of pipeline.

In the actual pipeline commissioning, before the crude oil is injected, hot water or other preheat medium is transfused into the cold pipeline. Thus, a lot of calories are brought into pipe, and the temperature of pipe and surrounding soil increase little by little. When the hot crude is injected after the preheating, the temperature difference among pipe, soil and hot crude is significantly reduced; meanwhile the crude heat loss is reduced. This preheat work provide the crude oil a safe temperature when it flows to the pipe ends [1].

The heat transfer involved in the preheating and commissioning process is not only between crude and pipe, but also between the preheat medium (hot water) and the around soil. Due to that the distance of a pipeline may reach hundreds of kilometers, and the heated pipe can influence the temperature of the soil with a range of $10 \mathrm{~m}$ around it [2], all these will bring huge amount of calculation and bring difficulties to the numerical simulation.

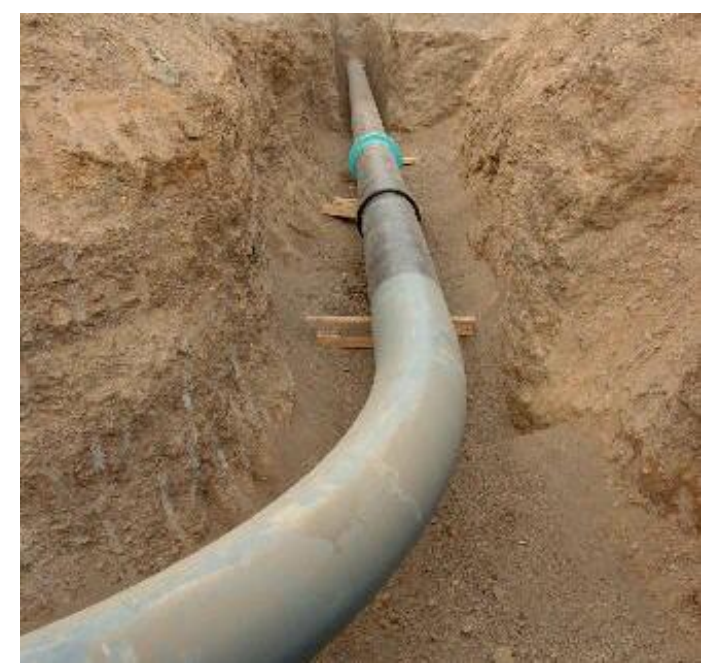

Fig. 1 Buried hot oil pipeline

\section{The Model and Spatial Discretization}

The heat transfer involved in the process of preheating and commissioning is among crude oil, metal pipe, pipe insulation layer, anti-corrosion layer, surrounding soil and air above the soil. All these above form the space model (Fig.2) of pipe preheating and commissioning.

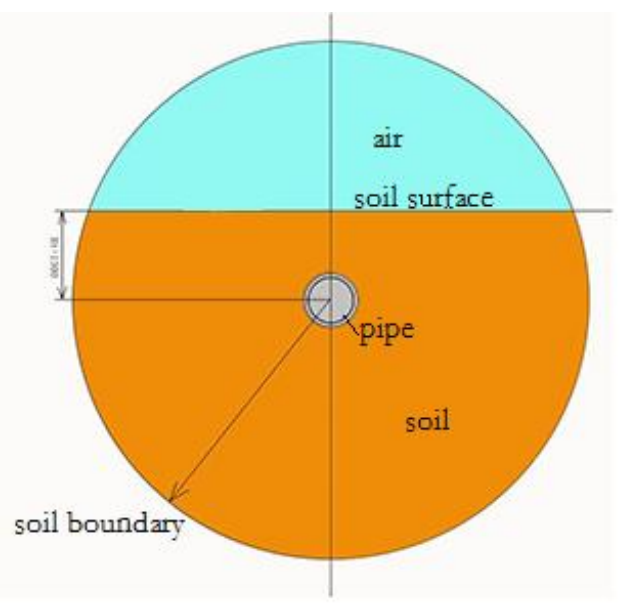

Fig. 2 The form of hot oil pipeline model 
Although the above model is relatively complete, however, it is too complex. Take a $60 \mathrm{~km}$ pipe section as an example, the pipe heat effect boundary is $10 \mathrm{~m}$ in diameter [2], and the average area of each grid is $0.001 \mathrm{~m}^{3}$, the whole model has more than $1.8 \times 10^{10}$ computing nodes. When simulating period of the pipeline is over 30 days and with a time step of 300 seconds, such a large amount of calculation is too much to the PC. As a result, the above model can't be widely used. In order to decrease the difficulty of pipeline numerical simulation, three kinds of technology is used to simplify the numerical simulation model.

\section{A. Pipeline discretization}

While in the process of pipeline preheating and putting into production, the medium temperature, the pipe temperature, the surrounding soil temperature vary in different locations along the pipe. Considering the pipe diameter, the buried depth, the temperature and the thermal conductivity are almost the same along the pipe axis, and the preheat medium and oil would be transported in the pipe in order, thus the temperature change of medium is continuous and gradual. The temperature of medium, pipe and surrounding soil in a cross section has small differences with their adjacent section, and the micro pipe section can be stood by the middle cross section. Therefore, the whole pipeline can be divided into several micro pipe sections [3]. Fig. 3 shows the micro pipeline sections and cross sections.

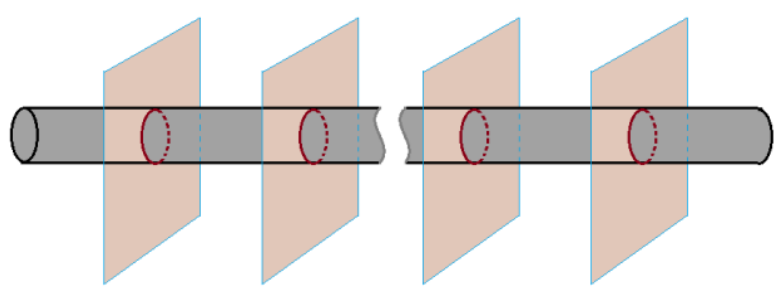

Fig. 3 The pipeline segment and cross section

\section{B. Simplification of the pipe cross sections}

As we can see in the Fig. 2, the pipe cross section is not rotational symmetric, but the horizontal symmetry with a central vertical line. When the pipe cross section is simulated, the left or the right half of cross section can be choose as the model, thus the calculation will be reduced by half [2].

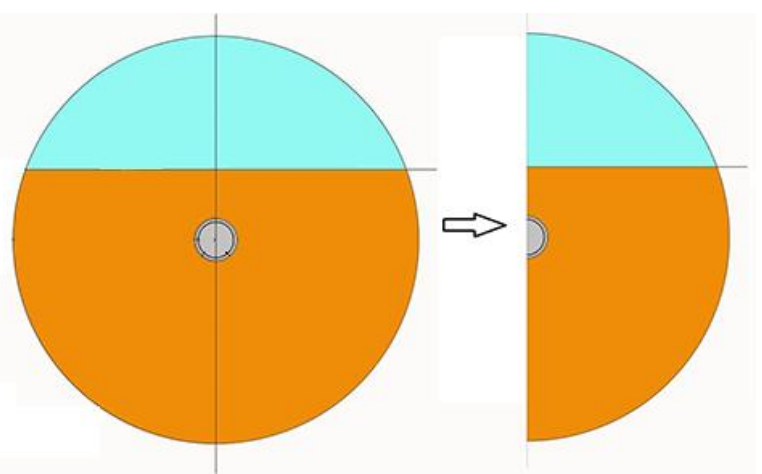

Fig. 4 The calculation area reduce
As in the Fig. 4, only the right half of the cross section is chosen, and the calculation can be reduced by half. And the pipe wall, the soil, near the central vertical line is set as the symmetry boundary, and the heat flux through the boundary is zero.

\section{The pipe cross-section spatial discretization}

As in the Fig. 4, the simulation space is a standard semicircle, so the pipe cross section discrete result is the structured polar grids. And the grids are shown in the left half of Fig. 5.
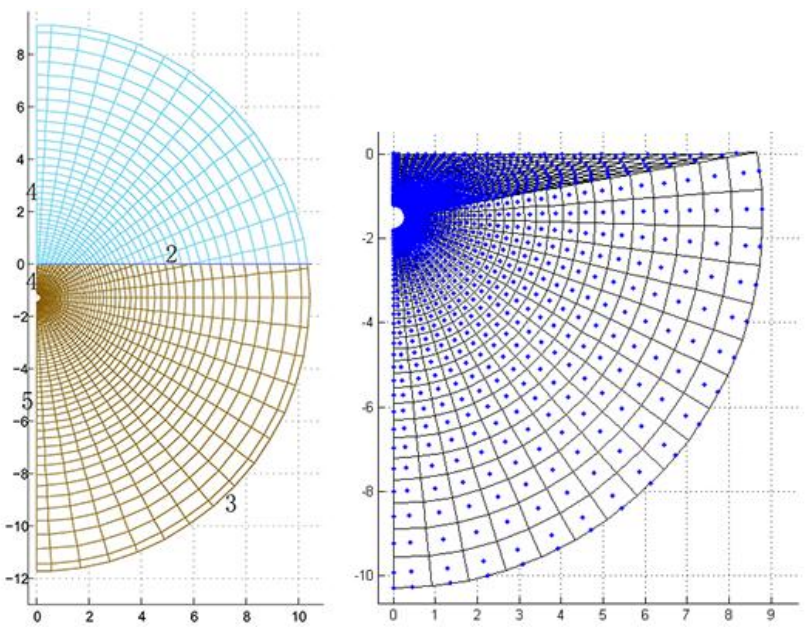

Fig. 5 The structured polar grids and its reform

But the structured polar grids applied here have two problems. First, the ground in the pipe cross section is a straight line, but in the discrete result, the ground line can't be replaced by the grids easily. Second, the grids above the ground line are not need in the numerical simulation, and they may waste storage space and computation time.

An improvement is made in the right half of Fig. 5, most of the grid notes above the pipeline is moved by a certain rule, and all the grid notes are under the ground line, and the up boundary is on the ground line. This reform not only solved the two above problems, but also improved the nodes intensity, and the calculation accuracy.

As shown in the right half of Fig. 5, most of the grid notes in the calculation region are similar to the note in the Fig. 6. The average parameters of each grid can be expressed by the parameters of $\mathrm{P}$, which is at the center of the grid. There are four adjacent grids around, named as N, S, W, and E. The distance between the nodes are represented by $\delta \mathrm{r}$ and $r \cdot \Delta \theta[2,3]$. 


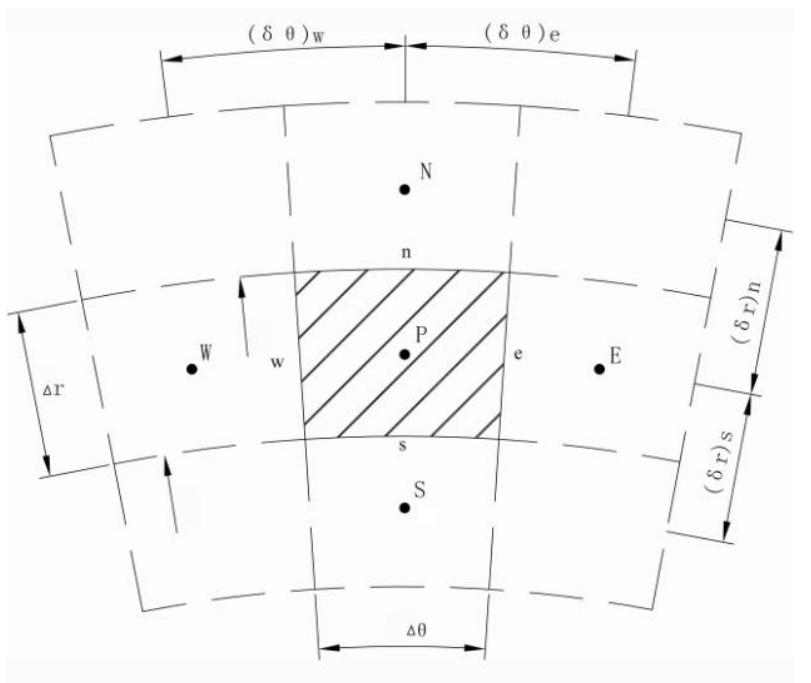

Fig. 6 Notes for the Finite Volume Method

For the heat transfer problem of polar coordinate grid, the heat conduction equation without inner heat source is set as the governing equation, as in (1) [4].

$$
\rho c \frac{\partial T}{\partial \tau}=\frac{1}{r} \frac{\partial}{\partial r}\left(\lambda r \frac{\partial T}{\partial r}\right)+\frac{1}{r^{2}} \frac{\partial}{\partial \varphi}\left(\lambda \frac{\partial T}{\partial \varphi}\right)
$$

Where

$\rho$ - The density of the material P.

$c$ - The capacity of the material P.

$\mathrm{T}$ - The temperature

$\tau$ - The time

$r$ - The diameter

$\lambda$ - The thermal conductivity

$\varphi$ - The angle

W, E, N, S - The signs of neighbor nodes

$\mathrm{w}, \mathrm{e}, \mathrm{n}, \mathrm{s}-$ The signs of surrounding interfaces

Finite volume method is used to discrete the governing equations, and the results as shown in equation $2[5,6]$.

$$
a_{P} T_{p}=a_{E} T_{E}+a_{W} T_{W}+a_{N} T_{N}+a_{S} T_{S}+b
$$

where

$$
\begin{aligned}
& a_{\mathrm{E}}=\frac{\Delta r}{r_{e}(\delta \theta)_{e} / \lambda_{n}} \ldots \ldots a_{\mathrm{W}}=\frac{\Delta r}{r_{w}(\delta \theta)_{w} / \lambda_{w}} \\
& a_{N}=\frac{r_{n} \Delta \theta}{(\delta r)_{n} / \lambda_{n}} \ldots \ldots a_{S}=\frac{r_{s} \Delta \theta}{(\delta r)_{s} / \lambda_{s}} \\
& a_{p}^{0}=\frac{(\rho c) \Delta V}{\Delta \tau} \\
& b=a_{p}^{0} T_{p}^{0} \\
& \Delta V=0.5\left(r_{n}+r_{s}\right) \Delta r \Delta \theta \\
& a_{P}=a_{E}+a_{W}+a_{N}+a_{S}+a_{p}^{0}
\end{aligned}
$$

The amount of the grids above the pipeline is not changed; the connections of adjacent grids are not changed, too. So the heat transfer relations are not changed, and (2) can also be used for the numerical calculation.

\section{Calculation Methods}

Although the physical model of the hot oil pipeline preheating and commissioning has been simplified by the three ways, the calculation amount is still huge. Good solution methods are also need to improve computational efficiency.

\section{A. Matrix computation}

Although the grid notes in the right side of Fig. 5 have been reformed, the grid number, arrangement did not change, the heat transfer between grids is not changed, too. So all the grid notes and their parameters can be stored and computed in matrix.

In every pipe cross sections, the semicircle can be divided into $\mathrm{m}$ shares, and there would be $\mathrm{M}=\mathrm{m}+2$ nodes. The radius from the inner pipe wall to the soil boundary can also be divided into $n$ shares, and there would be $\mathrm{N}=\mathrm{n}+2$ nodes. All the nodes in the computation area come to two-dimensional matrixes.

When the node parameters are stored in the matrix, the locations of node parameters in the matrixes are as the same as the location of real nodes in the computation area. All these give the conditions for the use of matrix calculation method. For example, the two-dimensional matrix $\mathrm{T}(\mathrm{M}, \mathrm{N})$ is used to store the nodes temperature, and other matrixes can also be used to store the density, capacity, thermal conductivity of mediums. All the parameters in the matrixes are as a whole when the thermal field is renewed.

For example, the temperature parameters in (2) can be expressed by the following matrixes.

$$
\begin{aligned}
& \mathrm{T}_{\mathrm{P}}=\mathrm{T}(2: \mathrm{M}-1,2: \mathrm{N}-1) \\
& \mathrm{T}_{\mathrm{E}}=\mathrm{T}(1: \mathrm{M}-2,2: \mathrm{N}-1) \\
& \mathrm{T}_{\mathrm{W}}=\mathrm{T}(3: \mathrm{M}, 2: \mathrm{N}-1) \\
& \mathrm{T}_{\mathrm{N}}=\mathrm{T}(2: \mathrm{M}-1,3: \mathrm{N}) \\
& \mathrm{T}_{\mathrm{S}}=\mathrm{T}(2: \mathrm{M}-1,1: \mathrm{N}-2)
\end{aligned}
$$

Other parameters like $a_{P}, a_{E}, a_{W}, a_{S}$, and $a_{N}$, which are the heat transfer coefficient between nodes, can also be expressed by the matrixes. The matrix computation in Matlab is optimized, and the computation time can greatly reduce by using matrix computation instead of circulation calculation, and the computational efficiency can also be improved.

\section{B. Parallel computing}

In the whole process of numerical simulation of pipeline preheating and commissioning, the calculation of renewing all the soil temperature field of cross sections takes most of the calculation time. As showing in Figure 3, the shapes and the grids in each across sections is similar, this creates the application condition for the parallel computing. 


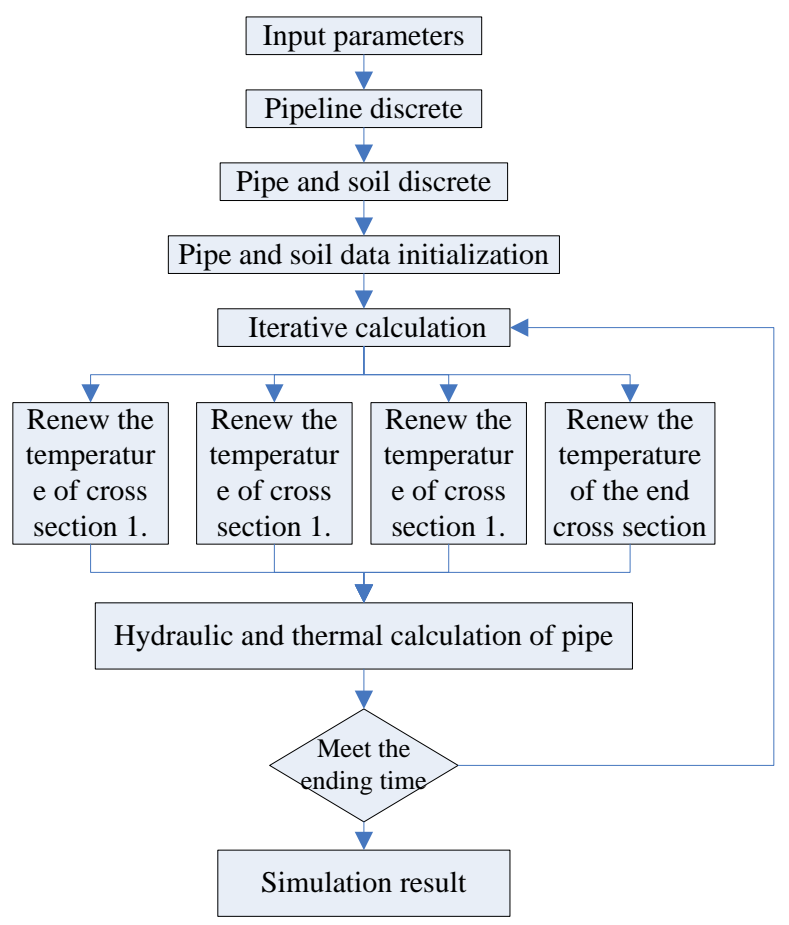

Fig. 7 The process map

As show in Fig.7, the numerical simulation of the hot oil pipeline preheating and commissioning process is connected in series. The most important processes of the simulation are the temperature field renewing and the hydraulic and thermal calculation, and the latter takes most of the calculation time. It's noticed that the temperature field renewing of one cross section is completely independence to other sections, and the renewing method for each section is similar. So the parallel computing method can be used in the temperature field renewing [7]. When a multi-core processor is used for calculation, the renewing of different cross section can be calculated with different cores, and the total calculation time can be saved.

\section{Result}

In order to verify the accuracy and efficiency of the parallel computing method and matrix calculation method in the numerical simulation of hot oil pipeline preheating and commissioning, an unstructured grid method and a serial computing method are used for compare. The computing environment and the result are as follow.

TABLE 1 Computer performance

\begin{tabular}{|c|c|}
\hline Items & parameter \\
\hline Program language & Matlab 2009a \\
\hline Processor amount & Quad-Core Processors \\
\hline CPU Frequency & $3.6 \mathrm{GHz}$ \\
\hline Memory & $8.0 \mathrm{~GB}$ \\
\hline Computer system & Win 7 64-bit system \\
\hline
\end{tabular}

TABLE 2 Parameters of pipeline and operation

\begin{tabular}{|c|c|}
\hline Items & parameter \\
\hline Pipe length & $100 \mathrm{~km}$ \\
\hline Pipe diameter & $323 \mathrm{~mm}$ \\
\hline Wall thickness & $6.7 \mathrm{~mm}$ \\
\hline Coating thickness & $3.0 \mathrm{~mm}$ \\
\hline Buried depth & $1.3 \mathrm{~m}$ \\
\hline Range of boundary & $10.0 \mathrm{~m}$ \\
\hline Preheating temperature & $70{ }^{\circ} \mathrm{C}$ \\
\hline Preheating flow speed & $0.8 \mathrm{~m} / \mathrm{s}$ \\
\hline Commission temperature & $70{ }^{\circ} \mathrm{C}$ \\
\hline Commission flow speed & $1.0 \mathrm{~m} / \mathrm{s}$ \\
\hline Soil and air temperature & $30{ }^{\circ} \mathrm{C}$ \\
\hline Soil density & $1700 \mathrm{~kg} / \mathrm{m}^{3}$ \\
\hline Soil capacity & $1900 \mathrm{~J} /\left(\mathrm{kg} \bullet{ }^{\circ} \mathrm{C}\right)$ \\
\hline Soil thermal conductivity & $0.6 \mathrm{~W} /\left(\mathrm{m} \bullet{ }^{\circ} \mathrm{C}\right)$ \\
\hline
\end{tabular}

TABLE 3 Discretization results of the cross section

\begin{tabular}{|c|c|c|}
\hline Items & Polar grids & $\begin{array}{c}\text { Delaunay } \\
\text { triangulation grids }\end{array}$ \\
\hline Nodes amount & $21 * 39=819$ & 572 \\
\hline Grids amount & $20 * 38=760$ & 1064 \\
\hline Grid interface & $\mathbf{2 1 * 3 8 + 2 0 * 3 9 = 1 5 7 8}$ & $\mathbf{1 5 9 6}$ \\
\hline Taken memory & $34 \mathrm{~kb}$ & $85 \mathrm{~kb}$ \\
\hline $\begin{array}{c}\text { Iteration } \\
\text { amount }\end{array}$ & 657 & 443 \\
\hline Iteration time & $2.13 \mathrm{~s}$ & $6.82 \mathrm{~s}$ \\
\hline
\end{tabular}

As shown in Table 3, the two grid discretization methods are solved iteratively to get the time independent numerical solution. The nodes amount of polar grids is 0.4 times more than the Delaunay triangulation grids, and the grids amount of polar grids is $70 \%$ as the Delaunay triangulation grids amount. The two set of grids have the same amount of grids interface. The Delaunay triangulation grids take more memory and iteration time.

TABLE 4 Computing time

\begin{tabular}{|c|c|c|c|}
\hline $\begin{array}{c}\text { Pipeline } \\
\text { length }\end{array}$ & $\begin{array}{c}\text { Serial computing } \\
\text { time }\end{array}$ & $\begin{array}{c}\text { Parallel } \\
\text { computing time }\end{array}$ & Proportion \\
\hline $\mathrm{km}$ & $\mathrm{s}$ & $\mathrm{s}$ & \\
\hline 40 & 508 & 144.5 & $28.4 \%$ \\
\hline 60 & 597.2 & 166.8 & $27.9 \%$ \\
\hline 80 & 666.4 & 178.1 & $26.7 \%$ \\
\hline 100 & 755.6 & 196.4 & $26.0 \%$ \\
\hline
\end{tabular}

Table 4 shows the computing time spent in the hot oil pipeline preheating and commissioning. When the pipeline goes longer, the both computing time is also longer. The parallel computing examples spent more than a quarter time 
spent by the serial computing examples.

The parallel computing method is much more efficiency than the serial computing method. The main reason is that the computing work is separated to each CPU processors, and the total costing time is separated, too.

A hot oil pipeline preheating and commissioning process is taken as an example, the pipe parameter is shown in the table 2. The numerical simulation result is shown in the following figures.

The Fig. 8 shows the pipe ends temperature change during the pipe preheating and commissioning. At the moment $\mathrm{A}$, hot water is injected in the pipeline, and at the moment $\mathrm{B}$, the pipeline full filled with the water. Then the water temperature at the end of the pipe is going higher. And at the moment $\mathrm{C}$, the water temperature is high enough for commission, and the hot oil is injected to replace the water. At the moment $\mathrm{D}$, the commission comes to the end. During the whole commission process, the crude oil temperature is still higher than the safety requirements.

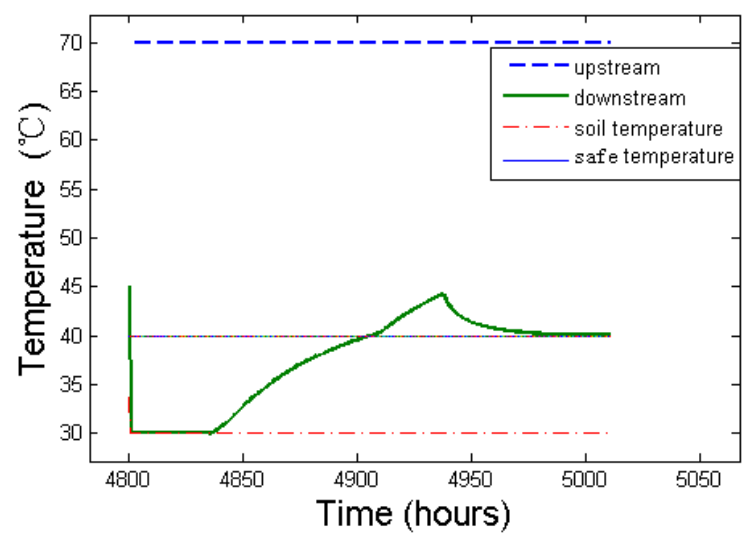

Fig 8 Ends temperature of the pipeline

The Fig.9 to the Fig.12 depict the temperature and heat flux during pipeline preheating process and commissioning process.

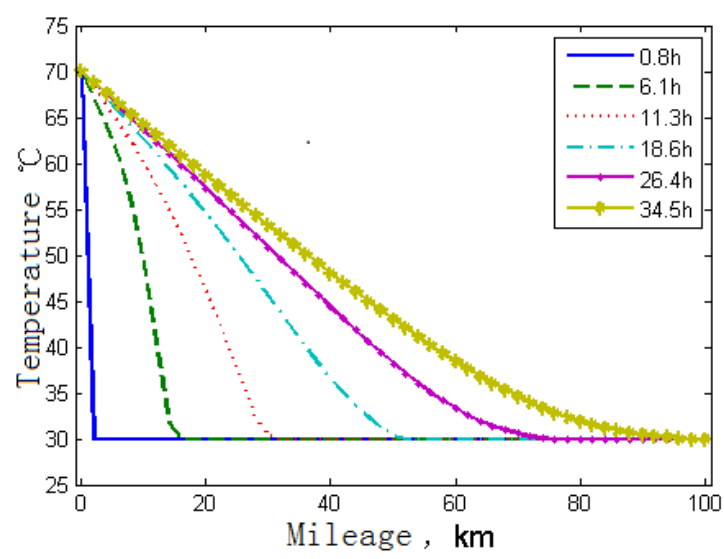

Fig. 9 The temperature profiles during water filling into the pipe

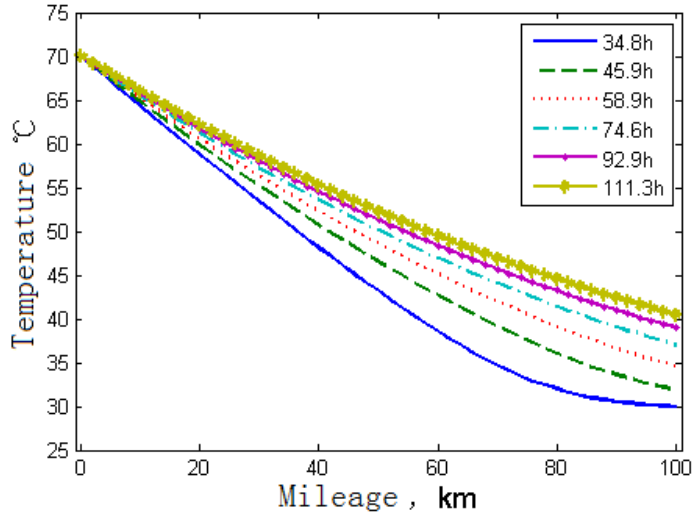

Fig. 10 The temperature profiles during preheating by hot water

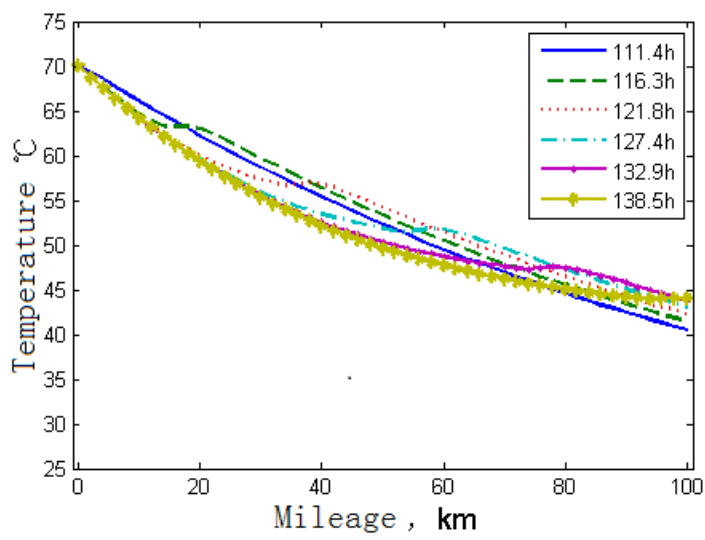

Fig. 11 The temperature profiles during oil filling into the pipe

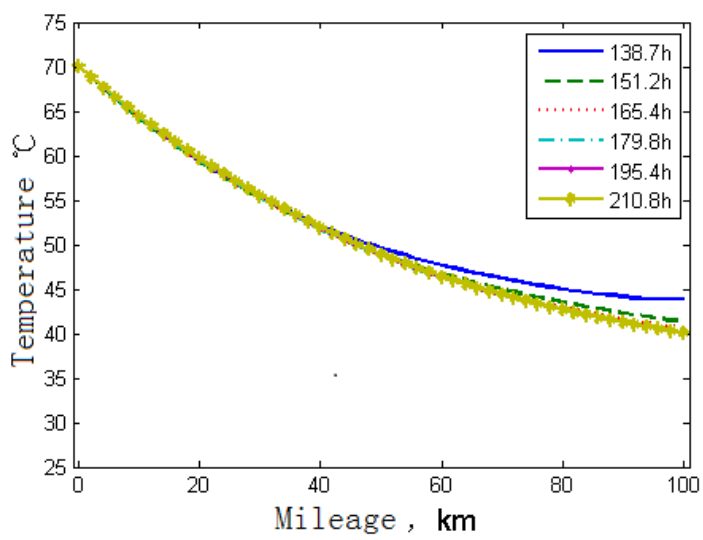

Fig. 12 The temperature profiles during commissioning

\section{Conclusions}

1. The pipe discretization method, the pipe section symmetrical treatment and the improved polar coordinate grid discretization method can simplify the physical model of hot oil pipeline. All these methods can reduce the grids amount, meanwhile improve the computing speed.

2. Because the pipe discretization and the polar coordinate grids create a good application condition, the 
matrix computations and parallel computing method are used in the numerical simulations. The two methods can significantly accelerate the computation speed, save the computational time, and improve the availability of the numerical simulation.

3. The simulation results of oil pipeline preheating and commissioning process show that the physical model and the computing methods can be effectively used.

\section{References}

[1] Xing, X., et al., Optimizing control parameters for crude pipeline preheating through numerical simulation. Applied Thermal Engineering, 2013. 51(1-2): p. 890-898.
[2] Yu, B., et al., Numerical simulation of a buried hot crude oil pipeline under normal operation. 2010. 30(17-18): p. 2670-2679.

[3] Yu, B., et al., Thermal impact of the products pipeline on the crude oil pipeline laid in one ditch - The effect of pipeline interval. 2008. 51(3-4): p. 597-609.

[4] Hakan Demir, A.K.G.T., Heat transfer of horizontal parallel pipe ground heat exchanger andexperimental verification, in Applied Thermal Engineering. 2009. p. 224-233.

[5] Wenquan Tao, Multi scale numerical simulation of fluid flow and heat transfer problems. 2009, Beijing: Science Press. P15-25

[6] Wenquan, Tao, Numerical Heat Transfer. 2002, Xi'an: Xi'an Jiao Tong University press. P566.

[7] Campbell, C., Miller, A., Paarallel Programming with Microsoft Visual C ++, Microsoft Press, 2012, ISBN: 978-0-7356-5175-3 